\title{
Treatment and Outcome of Congenital Adrenal Hyperplasia: 21-Hydroxylase Deficiency
}

\author{
Peter A. Lee, ${ }^{1,2}$ John S. Fuqua, ${ }^{1}$ and Todd D. Nebesio ${ }^{1}$ \\ ${ }^{1}$ James Whitcomb Riley Hospital for Children, Indiana University School of Medicine, Indianapolis, IN 46032, USA \\ ${ }^{2}$ Hershey Medical Center, Penn State University College of Medicine, Hershey, PA 17033, USA \\ Correspondence should be addressed to Peter A. Lee, leepa@iupui.edu
}

Received 31 December 2010; Accepted 31 December 2010

Copyright (C) 2010 Peter A. Lee et al. This is an open access article distributed under the Creative Commons Attribution License, which permits unrestricted use, distribution, and reproduction in any medium, provided the original work is properly cited.

The use of glucocorticoids to treat individuals with congenital adrenal hyperplasia $(\mathrm{CAH})$ was first reported by both Wilkins and Bartter in 1950. Since that time, the care of these patients has improved dramatically, and through the efforts of dedicated medical researchers, it continues to improve today. With early detection by newborn screening, initiation of treatment in infants with salt-wasting CAH has led to reduced morbidity and mortality. This special issue of the International Journal of Pediatric Endocrinology is intended to review the state of the art in medical treatment and psychological management as well as evaluations of the outcomes of patients with CAH.

We begin with a summary of the recent Endocrine Society Clinical Practice Guideline, which provides an overview of the diagnosis and treatment of CAH across the lifespan. We then move on to discussions concerning the diagnosis and treatment of nonclassic $\mathrm{CAH}$ and the management of the various forms of $\mathrm{CAH}$ in the adult patient. Although hydrocortisone has been the treatment of choice for many years in children with $\mathrm{CAH}$, more potent glucocorticoids such as dexamethasone are considered by some to be superior. A series of articles addresses the choice of glucocorticoid and the timing of administration. The section on treatment finishes with discussions of mineralocorticoid replacement and newer therapeutic modalities.

The next section deals with psychological management, specifically with regards to psychosocial stress in families of affected patients. We then move on to models of care provision, including establishment of a medical home, multidisciplinary care, and a discussion of the roles of support and advocacy groups in the care of individuals with CAH.
Assessments of treatment outcomes are critical for efforts to improve care. This issue finishes with a series of papers reviewing $\mathrm{CAH}$ outcomes, including growth in children, gynecologic and reproductive issues in women, bone health, and psychological, cognitive, and gender identity outcomes.

The guest editors hope that this issue will, in a small way, advance their understanding of $\mathrm{CAH}$ and stimulate additional research and multidisciplinary teamwork, with the goal of improving the lives of those affected with this potentially devastating condition.
Peter A. Lee John S. Fuqua Todd D. Nebesio 